\title{
Decoding and Recoding Signs and Images in the Wenxin Diaolong: from Title to Titles
}

文心雕龍的標諳, 圖像解碼和重新編碼

Simona Gallo 賈西媚

DoI: http://dx.doi.org/10.7359/825-2017-gall

Because meaning is an effect of relations and differences among signifiers along a potentially endless chain (polysemous, intertextual, subject to infinite linkages), it is always differential and deferred, never present as an original unity [...].

Lawrence Venuti

\section{Abstract}

The linguistic and cultural challenge that the translators of Liu Xie's masterpiece courageously accepted can be immediately perceivable within the Wenxin diaolong chapter titles, through which different approaches to translation - such as de-codification and re-codification of signs and images - come to existence. This contribution is meant to be a preliminary comparative study on the strategies adopted for the translation of the Wenxin diaolong chapter titles. First, a table of the original titles, followed by several translations into three European and alphabetic languages - namely Italian, English and French - has been created. The table, presented hereunder, allows for a multiperspective comparison, articulated into an unbiased discourse that takes into consideration the unavoidable shift in the architecture of the titles, as well as the semantic patterning and the lexical choices.

Keywords: Wenxin diaolong chapter titles, comparative study, approaches to translation, alphabetic languages, shift.

\section{摘要}

劉涩的代表作《文心雕龍》的譯者所面臨的語言與文化方面的挑戰從《文心雕龍》每個章節 的題目中可見一斑。因此需要通過不同的翻譯方法——例如通過編纂和重新編纂符號和圖 像——來展示。這篇文章是對《文心雕龍》章節標題所採用的翻譯策略的初步比較研究。 
首先是原始標題列表，接著是三種已經被譯成的歐洲語言一即義大利語、英語和法語。這 裡展示的表格, 可以進行不同視角的對比, 闡述了一種不帶偏見的話語, 並且考慮了標題 結構中不可避免的變化，以及語義模式和詞彙的選擇等因素。

關鍵詞：《文心雕龍》章節標題，對比研究，翻譯方法，字母語言，轉化。

\section{REFLECTIONS ON TRANSLATION}

In his reflection on the paradigm of translation, Paul Ricoeur (2004) mentioned that there are two main ways to conceive the act of translating, one strict, the other broad. Following Antoine Berman (1995), Ricoeur stated that translation may be interpreted as the rendering of a verbal message into another language, and this is the case of interlingual translation (Jakobson 1959); and recalling George Steiner (1997) for the broad sense, he said that any effort of intellectual understanding of the message's meaning can already be considered as an act of translation. The latter sense is summed by Steiner in the equation "understanding is translating" (ibid.), that in Ricoeur's interpretation can involve any intralingual translation and hermeneutic process.

A point of intersection of the two approaches may be read in Jiři Levýs declaration:

A translation is not a monistic composition, but an interpenetration and conglomerate of two structures. On the one hand, there are the semantic content and the formal contour of the original, on the other hand the entire system of aesthetic features bound up with the language of the translation. (Levý in Bassnett 2002, 16)

In her work, Translation Studies (Bassnett 2002), Susan Bassnett points out that translation is not merely a linguistic act, but also a semiotic one, whereby a process of decoding and recoding takes place (Bassnett 2002, 24). This sounds particularly true when taking into account two linguistic codes that do not share the same writing system, such as any European alphabetic language and Chinese. Therefore, the issue of decoding and recoding what is densely expressed by what sinologist Léon Vandermeersch (2013) calls a "graphic language" may raise the problem of the (un)translatability of the wen 文, before its inherent meaning. From this point of view, since the graphic aspect of a character cannot be reproduced, we could assume that there is an unbridgeable gap between the codes, which translation is unable to solve. Conscious of all this, the translators of the Wenxin diaolong 
worked on the "constitutive shift" ${ }^{1}$ : committed to recreating the icastic power of the wen 文, they noticeably spent great efforts transcending the paradox of equivalence ${ }^{2}$ without identity (Ricoeur 2004, 14) or even the fact that "there can be no fully exact translations. Hence, there can be no fully exact translation" (Nida 2012, 126). By doing so, they overtly accepted the challenge to translate the linguistic and cultural (un)translatable.

\section{2. "WenXin DiAOLONG" CHAPTER TITLES}

One of the perspectives through which this can be demonstrated is the one focusing on chapter titles, only two sinograms artfully selected by Liu Xie to concentrate the meaning of the entire chapter. This contribution takes into consideration chapter titles and the different strategies adopted by several well-known translators to re-code them from Chinese.

The table below (see Tab. 1) lists in comparison the original Chinese version of the fifty chapters of the Wenxin diaolong with some of its best-known and more representative translations into three European languages, specifically into Italian, English and French, and namely:

A. Italian version, translated by Alessandra Cristina Lavagnino, entitled Il tesoro delle lettere. Un intaglio di dragbi (Liu Xie 1995);

B. English version, translated by Vincent Yu-chung Shih, entitled The Literary Mind and the Carving of Dragons (Liu Xie 1959);

C. English version, translated by Siu-kit Wong, Allan Chung-han Lo, Kwong-tai Lam, entitled The Book of Literary Design (Liu Xie 1999);

D. English version, translated by Yang Guobin, entitled The Literary Mind and the Carving of Dragons (Liu Xie 2003);

E. French version, translated by Chen Shuyu, entitled L'essence de la littérature et la gravure de dragons (Liu Xie 2010).

As this table was elaborated by the current author on the occasion of the International Symposium “Wenxin Duibua 文心對話 - Dialogue on The Literary Mind / The Core of Writing" ", held at the University of Milan in May 2014, I chose to include the Italian column (Liu Xie 1995) first.

1 Defined by Popovič as "an inevitable shift that takes place as a result of differences between two languages, two poetics and two styles” (Bassnett 2002, 142).

2 Along with Popovič definition of "equivalence” (Bassnett 2002, 33).

3 Organized by the Department of Studies in Language Mediation and Intercultural Communication and the Confucius Institute of the University of Milan. The integrity of the original wording has been maintained. 
Table 1

\begin{tabular}{|c|c|c|c|c|c|c|}
\hline & $\begin{array}{l}\text { 文心 } \\
\text { 雕龍 }\end{array}$ & (Liu Xie 1995) & (Liu Xie 1959) & (Liu Xie 1999) & (Liu Xie 2003) & (Liu Xie 2010) \\
\hline 1 & 原道 & $\begin{array}{l}\text { L'Origine } \\
\text { nel Dao }\end{array}$ & $\begin{array}{l}\text { On Tao, } \\
\text { the source }\end{array}$ & $\begin{array}{l}\text { The Way } \\
\text { the Origin }\end{array}$ & $\begin{array}{l}\text { Tracing } \\
\text { the Origin } \\
\text { to the Dao }\end{array}$ & $\begin{array}{l}\text { Yuan Dao- } \\
\text { L'origine } \\
\text { de l'écriture }\end{array}$ \\
\hline 2 & 徵聖 & $\begin{array}{c}\text { Le } \\
\text { testimonianze } \\
\text { dei Saggi }\end{array}$ & $\begin{array}{c}\text { Evidence } \\
\text { from the Sage }\end{array}$ & $\begin{array}{l}\text { The Sages } \\
\text { the Oracle }\end{array}$ & $\begin{array}{l}\text { Venerating } \\
\text { the Sages }\end{array}$ & $\begin{array}{c}\text { Zheng Sheng - } \\
\text { Les critères } \\
\text { des sages }\end{array}$ \\
\hline 3 & 宗經 & $\begin{array}{l}\text { La deferenza } \\
\text { ai Classici }\end{array}$ & $\begin{array}{c}\text { The Classics } \\
\text { as literary } \\
\text { sources }\end{array}$ & $\begin{array}{l}\text { The Classics } \\
\text { the Forefather }\end{array}$ & $\begin{array}{c}\text { Modelling } \\
\text { on the Classics }\end{array}$ & $\begin{array}{l}\text { ZongJing - } \\
\text { Suivre } \\
\text { les classiques }\end{array}$ \\
\hline 4 & 正緯 & $\begin{array}{c}\text { Corretta lettura } \\
\text { degli Apocrifi }\end{array}$ & $\begin{array}{l}\text { Emendation } \\
\text { of Apocrypha }\end{array}$ & $\begin{array}{l}\text { Putting } \\
\text { the Cabala } \\
\text { in Order }\end{array}$ & $\begin{array}{c}\text { A Proper } \\
\text { Understanding } \\
\text { of Apocrypha }\end{array}$ & $\begin{array}{c}\text { Zheng Wei- } \\
\text { La correction } \\
\text { des apocryphes }\end{array}$ \\
\hline 5 & 辯騷 & $\begin{array}{l}\text { I giudizi } \\
\text { sul "Li Sao" }\end{array}$ & $\begin{array}{c}\text { An analysis } \\
\text { of Sao }\end{array}$ & $\begin{array}{l}\text { Isolating } \\
\text { Sao Poetry }\end{array}$ & $\begin{array}{c}\text { Evaluating Sao, } \\
\text { or the Songs } \\
\text { of the South }\end{array}$ & $\begin{array}{c}\text { Bian Sao- } \\
\text { L'évaluation } \\
\text { des élégies } \\
\text { de Chu }\end{array}$ \\
\hline 6 & 明詩 & $\begin{array}{l}\text { Illustrazione } \\
\text { della poesia }\end{array}$ & $\begin{array}{l}\text { An exegesis } \\
\text { of poetry }\end{array}$ & $\begin{array}{l}\text { Understanding } \\
\text { Shi Poetry }\end{array}$ & $\begin{array}{l}\text { Illuminating } \\
\text { Poetry }\end{array}$ & $\begin{array}{c}\text { Ming Shi- } \\
\text { Le traitement } \\
\text { des poèmes }\end{array}$ \\
\hline 7 & 樂府 & Yue fu & $\begin{array}{c}\text { Musical poetry } \\
\quad(y \ddot{u} e h-f u)\end{array}$ & $\begin{array}{l}\text { The Yuefu } \\
\text { Poems }\end{array}$ & $\begin{array}{c}\text { Yuefu, } \\
\text { or Poems } \\
\text { of the "Music } \\
\text { Bureau" }\end{array}$ & $\begin{array}{l}Y u e F u- \\
\text { Le poème } \\
\text { chanté }\end{array}$ \\
\hline 8 & 銓賦 & $\begin{array}{l}\text { La verità } \\
\text { sul } f u\end{array}$ & $\begin{array}{l}\text { Elucidation } \\
\quad \text { of } f u\end{array}$ & $\begin{array}{l}\text { Explaining } \\
\text { Fu Poetry }\end{array}$ & $\begin{array}{c}\text { Interpreting } \\
F u \text {, or } \\
\text { Rhyme-prose }\end{array}$ & $\begin{array}{c}\text { Quan Fu- } \\
\text { L'interprétation } \\
\text { du fu }\end{array}$ \\
\hline 9 & 頌讚 & Song e zan & $\begin{array}{c}\text { Ode and } \\
\text { Pronouncement } \\
\text { (the sung } \\
\text { and the tsan) }\end{array}$ & $\begin{array}{c}\text { Eulogistic } \\
\text { Songs } \\
\text { and Summaries }\end{array}$ & $\begin{array}{c}\text { Hymn } \\
\text { and Eulogy }\end{array}$ & $\begin{array}{l}\text { Song Zan - } \\
\text { L'éloge } \\
\text { et le conseil }\end{array}$ \\
\hline 10 & 祝盟 & $\begin{array}{l}\text { Invocazione } \\
\text { agli spiriti } \\
\text { e alleanza } \\
\text { solenne }\end{array}$ & $\begin{array}{l}\text { Sacrifical prayer } \\
\text { and Oath } \\
\text { of agreement } \\
\text { (the } c h u \\
\text { and the meng) }\end{array}$ & $\begin{array}{l}\text { Prayers } \\
\text { and Oaths }\end{array}$ & $\begin{array}{c}\text { Prayer } \\
\text { and Oath }\end{array}$ & $\begin{array}{l}\text { Zhu Meng - } \\
\text { Les prières } \\
\text { des vœux } \\
\text { et les serments }\end{array}$ \\
\hline 11 & 銘箴 & $\begin{array}{c}\text { Epigrafe } \\
\text { e ammonizione }\end{array}$ & $\begin{array}{c}\text { Inscription } \\
\text { and exhortation } \\
\text { (the ming } \\
\text { and the chen) }\end{array}$ & $\begin{array}{l}\text { Inscriptions } \\
\text { and } \\
\text { Punctures }\end{array}$ & $\begin{array}{l}\text { Inscription } \\
\text { and } \\
\text { Admonition }\end{array}$ & $\begin{array}{c}\text { Ming Zhen- } \\
\text { L'inscription } \\
\text { et } \\
\text { l'avertissement }\end{array}$ \\
\hline 12 & 昩碑 & $\begin{array}{l}\text { Elogio } \\
\text { funebre } \\
\text { e stele }\end{array}$ & $\begin{array}{c}\text { Elegy and Stone } \\
\text { inscription } \\
\text { (the lei } \\
\text { and the pei) }\end{array}$ & $\begin{array}{l}\text { Laudations } \\
\text { and Epitaphs }\end{array}$ & $\begin{array}{l}\text { Mourning- } \\
\text { Song } \\
\text { and Epitaph }\end{array}$ & $\begin{array}{c}\text { Lei Bei - } \\
\text { L'élégie } \\
\text { et l'inscription } \\
\text { lapidaire }\end{array}$ \\
\hline
\end{tabular}




\begin{tabular}{|c|c|c|c|c|c|c|}
\hline & $\begin{array}{l}\text { 文心 } \\
\text { 雕龍 }\end{array}$ & (Liu Xie 1995) & (Liu Xie 1959) & (Liu Xie 1999) & (Liu Xie 2003) & (Liu Xie 2010) \\
\hline 13 & 哀帅 & $\begin{array}{l}\text { Compianto } \\
\text { e ode funebre }\end{array}$ & $\begin{array}{l}\text { Lament } \\
\text { and } \\
\text { Condolence } \\
\text { (the } a i \\
\text { and the } t a o \text { ) }\end{array}$ & $\begin{array}{c}\text { Commiseration } \\
\text { and } \\
\text { Condolence }\end{array}$ & $\begin{array}{l}\text { Lament } \\
\text { and } \\
\text { Condolence }\end{array}$ & $\begin{array}{c}\text { Ai Diao- } \\
\text { La complainte } \\
\text { et les } \\
\text { condoléances }\end{array}$ \\
\hline 14 & 雜文 & $\begin{array}{c}\text { Forme diverse } \\
\text { [Replica, Sette, } \\
\text { Collana } \\
\text { di perle, ecc.] }\end{array}$ & $\begin{array}{l}\text { Miscellaneous } \\
\text { writings }\end{array}$ & $\begin{array}{l}\text { Miscellaneous } \\
\text { Rythmic Prose }\end{array}$ & $\begin{array}{c}\text { Miscellaneous } \\
\text { Writings }\end{array}$ & $\begin{array}{l}Z a \text { Wen- } \\
\text { Les écrits } \\
\text { divers }\end{array}$ \\
\hline 15 & 諧謡 & $\begin{array}{c}\text { Facezia } \\
\text { ed enigma }\end{array}$ & $\begin{array}{l}\text { Humor } \\
\text { and Enigma } \\
\text { (the bsieb } \\
\text { and the yin) }\end{array}$ & $\begin{array}{c}\text { Puns } \\
\text { and Parables }\end{array}$ & $\begin{array}{c}\text { Jesting Rhymes } \\
\text { and Puzzles }\end{array}$ & $\begin{array}{c}\text { Xie Yin- } \\
\text { L'humour } \\
\text { et la métaphore }\end{array}$ \\
\hline 16 & 史傳 & $\begin{array}{l}\text { Storiografia } \\
\text { e Commento, } \\
\text { o biografia }\end{array}$ & $\begin{array}{l}\text { Historical } \\
\text { writings }\end{array}$ & $\begin{array}{c}\text { Scribal } \\
\text { Hermeneutics }\end{array}$ & $\begin{array}{l}\text { Historical } \\
\text { Writings }\end{array}$ & $\begin{array}{l}\text { Shi Zhuan- } \\
\text { Les écrits } \\
\text { historiques }\end{array}$ \\
\hline 17 & 諸子 & $\begin{array}{c}\text { Le "Opere } \\
\text { dei Maestri" }\end{array}$ & $\begin{array}{l}\text { Speculative } \\
\text { writings }\end{array}$ & $\begin{array}{c}\text { The } \\
\text { Philosophers }\end{array}$ & $\begin{array}{c}\text { Philosophical } \\
\text { Writings }\end{array}$ & $\begin{array}{l}Z \text { Zhu } Z i- \\
\text { Les écrits } \\
\text { des grand } \\
\text { penseurs }\end{array}$ \\
\hline 18 & 論說 & $\begin{array}{c}\text { Lun } \\
\text { e onorazione }\end{array}$ & $\begin{array}{l}\text { Treatise } \\
\text { and } \\
\text { Discussion } \\
\text { (the lun } \\
\text { and the shuo) }\end{array}$ & $\begin{array}{l}\text { Argument } \\
\text { and } \\
\text { Persuasion }\end{array}$ & $\begin{array}{c}\text { The Treatise } \\
\text { and } \\
\text { the Speech }\end{array}$ & $\begin{array}{l}\text { Lun Shuo- } \\
\text { Les essais } \\
\text { et les discours }\end{array}$ \\
\hline 19 & 詔策 & $\begin{array}{l}\text { Proclami } \\
\text { e decreti }\end{array}$ & $\begin{array}{c}\text { Edict } \\
\text { and Script } \\
\text { (the chao } \\
\left.\text { and the } t s^{\prime} e\right)\end{array}$ & $\begin{array}{c}\text { Imperial } \\
\text { Edicts }\end{array}$ & $\begin{array}{c}\text { The Edict } \\
\text { and the Decree } \\
\text { of Enfeoffment }\end{array}$ & $\begin{array}{l}\text { Zhao Ce- } \\
\text { Les décrets } \\
\text { et les rapports } \\
\text { impériaux }\end{array}$ \\
\hline 20 & 檄移 & \begin{tabular}{|c|} 
Dichiarazioni \\
di guerre \\
e proclama \\
di riprensione
\end{tabular} & $\begin{array}{c}\text { War } \\
\text { proclamation } \\
\text { and Dispatch } \\
\text { (the } b s i \\
\text { and the } i \text { ) }\end{array}$ & $\begin{array}{c}\text { Declaration } \\
\text { of War } \\
\text { and of Disquiet }\end{array}$ & $\begin{array}{l}\text { The War } \\
\text { Proclamation } \\
\text { and the } \\
\text { Dispatch }\end{array}$ & $\begin{array}{c}X i Y i- \\
\text { L'appel } \\
\text { au combat } \\
\text { et la lettre } \\
\text { officielle }\end{array}$ \\
\hline 21 & 封禪 & $\begin{array}{l}\text { Sacrifici } \\
\text { al Cielo } \\
\text { e sacrifici } \\
\text { alla Terra }\end{array}$ & $\begin{array}{l}\text { Sacrifices } \\
\text { to Heave } \\
\text { and Earth } \\
\text { (feng shan) }\end{array}$ & $\begin{array}{c}\text { The Fengshan } \\
\text { Sacrifice }\end{array}$ & $\begin{array}{c}\text { The Sacrificial } \\
\text { Address } \\
\text { to Heaven } \\
\text { and Earth }\end{array}$ & $\begin{array}{c}\text { Feng Shan- } \\
\text { Les écrits } \\
\text { des sacrifices } \\
\text { au Ciel } \\
\text { et à la Terre }\end{array}$ \\
\hline 22 & 章表 & $\begin{array}{c}\text { Memoriali } \\
\text { di } \\
\text { ringraziamento } \\
\text { e di richiesta }\end{array}$ & $\begin{array}{c}\text { Memorial, } \\
\text { part I } \\
\text { (the chang } \\
\text { and the piao) }\end{array}$ & $\begin{array}{c}\text { Memorials } \\
\text { Illuminative } \\
\text { and } \\
\text { Manifestative }\end{array}$ & \begin{tabular}{|c|} 
Laudatory \\
Address \\
and Statement \\
on Government \\
Affairs
\end{tabular} & $\begin{array}{l}\text { Zhang Biao - } \\
\text { Les mémoires } \\
\text { impériaux (I) }\end{array}$ \\
\hline
\end{tabular}




\begin{tabular}{|c|c|c|c|c|c|c|}
\hline & $\begin{array}{l}\text { 文心 } \\
\text { 雕龍 }\end{array}$ & (Liu Xie 1995) & (Liu Xie 1959) & (Liu Xie 1999) & (Liu Xie 2003) & (Liu Xie 2010) \\
\hline 23 & 奏啟 & Memoriali & $\begin{array}{l}\text { Memorial, } \\
\text { part II } \\
\text { (the tsou } \\
\text { and the } c b^{\prime} i \text { ) }\end{array}$ & $\begin{array}{c}\text { Reports } \\
\text { to the Throne } \\
\text { and Statements }\end{array}$ & $\begin{array}{c}\text { Report } \\
\text { and } \\
\text { Memorandum }\end{array}$ & $\begin{array}{c}\text { Zou Qi- } \\
\text { Les mémoires } \\
\text { impériaux (II) }\end{array}$ \\
\hline 24 & 議對 & $\begin{array}{l}\text { Opinione } \\
\text { e replica }\end{array}$ & $\begin{array}{c}\text { Discussion } \\
\text { and Answer } \\
\text { (the } i \\
\text { and the } t u i)\end{array}$ & $\begin{array}{c}\text { Debates } \\
\text { and Answers }\end{array}$ & $\begin{array}{l}\text { The Discussion } \\
\text { and the } \\
\text { Examination } \\
\text { Essay }\end{array}$ & $\begin{array}{c}Y i D u i- \\
\text { La discussion } \\
\text { et la réponse }\end{array}$ \\
\hline 25 & 書記 & $\begin{array}{l}\text { La lettera } \\
\text { [e altre forme } \\
\text { minori] }\end{array}$ & $\begin{array}{l}\text { Epistolary } \\
\text { writing } \\
\text { (the } s b u \\
\text { and the } c b i)\end{array}$ & $\begin{array}{c}\text { Notes } \\
\text { and Letters }\end{array}$ & $\begin{array}{c}\text { Epistolary } \\
\text { Writing } \\
\text { and } \\
\text { Miscellaneous } \\
\text { Records }\end{array}$ & $\begin{array}{c}\text { ShuJi- } \\
\text { La } \\
\text { correspondance } \\
\text { e le rapport } \\
\text { administratif }\end{array}$ \\
\hline 26 & 神思 & $\begin{array}{l}\text { Il pensiero } \\
\text { poetico }\end{array}$ & $\begin{array}{c}\text { Spiritual } \\
\text { thought } \\
\text { or Imagination } \\
(s b e n-s s u)\end{array}$ & $\begin{array}{c}\text { Magical } \\
\text { Imagination }\end{array}$ & $\begin{array}{c}\text { Shensi, } \\
\text { or Imagination }\end{array}$ & $\begin{array}{c}\text { Shen } S i- \\
\text { La pensée } \\
\text { et } \\
\text { l'imagination }\end{array}$ \\
\hline 27 & 體性 & $\begin{array}{c}\text { Stile } \\
\text { e personalità }\end{array}$ & $\begin{array}{c}\text { Style } \\
\text { and Nature } \\
\left(t^{\prime} i \text {-bsing }\right)\end{array}$ & $\begin{array}{c}\text { Style } \\
\text { and } \\
\text { Personality }\end{array}$ & $\begin{array}{c}\text { Style } \\
\text { and Natural } \\
\text { Endowments }\end{array}$ & $\begin{array}{l}\text { Ti Xing- } \\
\text { Les genres } \\
\text { littéraires } \\
\text { et la nature } \\
\text { de l'auteur }\end{array}$ \\
\hline 28 & 風骨 & $\begin{array}{l}\text { "Vento" } \\
\text { e "ossa" }\end{array}$ & $\begin{array}{l}\text { The wind } \\
\text { and the bone } \\
\text { (feng-ku) }\end{array}$ & $\begin{array}{c}\text { The Affective } \\
\text { Air } \\
\text { and the } \\
\text { Literary Bones }\end{array}$ & $\begin{array}{c}\text { "Wind" } \\
\text { and "Bone" }\end{array}$ & $\begin{array}{l}\text { Feng Gu- } \\
\text { Le charme } \\
\text { et l'essence }\end{array}$ \\
\hline 29 & 通變 & $\begin{array}{l}\text { Continuità e } \\
\text { mutamento }\end{array}$ & $\begin{array}{c}\text { Flexible } \\
\text { adaptability } \\
\text { to varying } \\
\text { situations } \\
\text { (t'ung-pien) }\end{array}$ & $\begin{array}{l}\text { Continuity } \\
\text { and Change }\end{array}$ & $\begin{array}{l}\text { Continuity } \\
\text { and Change }\end{array}$ & $\begin{array}{c}\text { Tong Bian- } \\
\text { Comprendre } \\
\text { le changement }\end{array}$ \\
\hline 30 & 定勢 & $\begin{array}{l}\text { Definizione } \\
\text { della maniera }\end{array}$ & $\begin{array}{l}\text { On choice } \\
\text { of style }\end{array}$ & Stylistic Force & $\begin{array}{l}\text { Choosing } \\
\text { the Style, } \\
\text { or Natural } \\
\text { Tendency }\end{array}$ & $\begin{array}{l}\text { Ding Sbi- } \\
\text { Le choix } \\
\text { du style }\end{array}$ \\
\hline 31 & 情采 & $\begin{array}{l}\text { Emozioni } \\
\text { e bellezza }\end{array}$ & $\begin{array}{l}\text { Emotion } \\
\text { and Literary } \\
\text { expression }\end{array}$ & $\begin{array}{l}\text { Substance } \\
\text { and Style }\end{array}$ & $\begin{array}{l}\text { Feeling } \\
\text { and Art }\end{array}$ & $\begin{array}{l}\text { Qing Cai - } \\
\text { Les émotions et } \\
\text { les expressions } \\
\text { littéraires }\end{array}$ \\
\hline 32 & 鎔裁 & $\begin{array}{l}\text { Gettare } \\
\text { in forma } \\
\text { e tagliare }\end{array}$ & $\begin{array}{l}\text { Casting } \\
\text { and cutting, } \\
\text { or, on editing } \\
\text { of ideas } \\
\text { and rhetoric } \\
\text { (jung-ts'ai) }\end{array}$ & $\begin{array}{c}\text { Modelling } \\
\text { and Tailoring }\end{array}$ & $\begin{array}{c}\text { Casting } \\
\text { and Cutting }\end{array}$ & $\begin{array}{l}\text { Rong Cai- } \\
\text { Les détails } \\
\text { et la concision }\end{array}$ \\
\hline
\end{tabular}




\begin{tabular}{|c|c|c|c|c|c|c|}
\hline & $\begin{array}{l}\text { 文心 } \\
\text { 雕龍 }\end{array}$ & (Liu Xie 1995) & (Liu Xie 1959) & (Liu Xie 1999) & (Liu Xie 2003) & (Liu Xie 2010) \\
\hline 33 & 聲律 & $\begin{array}{l}\text { Le regole } \\
\text { tonali }\end{array}$ & Musicalness & The Music & Prosody & $\begin{array}{l}\text { Sheng L L̈̈- } \\
\text { Le ton } \\
\text { et la rhyme }\end{array}$ \\
\hline 34 & 章句 & $\begin{array}{c}\text { Paragrafo / } \\
\text { strofa e frase / } \\
\text { verso }\end{array}$ & $\begin{array}{c}\text { Paragraph } \\
\text { and Sentence }\end{array}$ & $\begin{array}{l}\text { Chapter } \\
\text { and Verse }\end{array}$ & $\begin{array}{c}\text { Paragraph } \\
\text { and Sentence }\end{array}$ & $\begin{array}{l}\text { Zang Ju- } \\
\text { Le paragraphe } \\
\text { et a phrase }\end{array}$ \\
\hline 35 & 麗辭 & Parallelismi & $\begin{array}{l}\text { Linguistic } \\
\text { parallelism }\end{array}$ & Couplets & Parallelism & $\begin{array}{c}\mathrm{LiCi}- \\
\text { L'art } \\
\text { du parallélisme }\end{array}$ \\
\hline 36 & 比興 & $\begin{array}{l}\text { Paragone } \\
\text { e xing }\end{array}$ & $\begin{array}{l}\text { Metaphor and } \\
\text { Allegory } \\
\text { (pi and bsing) }\end{array}$ & $\begin{array}{l}B i \text { and Xing - } \\
\text { Two Types } \\
\text { of Metaphor }\end{array}$ & $\begin{array}{l}\text { Comparison } \\
\text { and Metaphor }\end{array}$ & $\begin{array}{l}\text { Bi Xing- } \\
\text { La comparaison } \\
\text { et la métaphore }\end{array}$ \\
\hline 37 & 誇飾 & L'iperbole & $\begin{array}{l}\text { Embellishment } \\
\text { as description }\end{array}$ & Hyperbole & Hyperbole & $\begin{array}{l}\text { Kua Shi- } \\
\text { L’hyperbole }\end{array}$ \\
\hline 38 & 事類 & $\begin{array}{l}\text { Esempi } \\
\text { e citazioni }\end{array}$ & $\begin{array}{c}\text { Factual allusion } \\
\text { and textual } \\
\text { reference }\end{array}$ & Allusions & Allusions & $\begin{array}{l}\text { Shi Lei- } \\
\text { La citation }\end{array}$ \\
\hline 39 & 練字 & $\begin{array}{l}\text { La scelta } \\
\text { dei caratteri }\end{array}$ & $\begin{array}{l}\text { Philology } \\
\text { and Choice } \\
\text { of words }\end{array}$ & $\begin{array}{l}\text { Language } \\
\text { Refinement }\end{array}$ & $\begin{array}{l}\text { Choosing } \\
\text { the Right } \\
\text { Word }\end{array}$ & $\begin{array}{l}\text { Lian } Z i- \\
\text { Le choix } \\
\text { des mots }\end{array}$ \\
\hline 40 & 隱秀 & $\begin{array}{l}\text { Il recondito } \\
\text { e l'eccellente }\end{array}$ & $\begin{array}{c}\text { The recondite } \\
\text { and } \\
\text { the conspicuous } \\
(y i n-b s i u)\end{array}$ & $\begin{array}{l}\text { Hidden Grace } \\
\text { and the Visible } \\
\text { Flower }\end{array}$ & $\begin{array}{l}\text { Concealed } \\
\text { and Evident } \\
\text { Excellence }\end{array}$ & $\begin{array}{c}\text { Yin Xiu- } \\
\text { L'implicite } \\
\text { et l'aphorisme }\end{array}$ \\
\hline 41 & 指瑕 & $\begin{array}{l}\text { Denuncia } \\
\text { dei difetti }\end{array}$ & Literary flaws & $\begin{array}{l}\text { Picking out } \\
\text { the } \\
\text { Imperfections }\end{array}$ & $\begin{array}{c}\text { Flaws } \\
\text { in Writing }\end{array}$ & $\begin{array}{c}\text { Zhi Xia- } \\
\text { Lindication } \\
\text { des erreurs }\end{array}$ \\
\hline 42 & 養氣 & $\begin{array}{l}\text { Come } \\
\text { alimentare } \\
\text { il } q i\end{array}$ & $\begin{array}{c}\text { The nourishing } \\
\text { of vitality }\end{array}$ & $\begin{array}{l}\text { Preservation } \\
\text { of the } \\
\text { Life-breath }\end{array}$ & $\begin{array}{c}\text { Nourishing } \\
\text { qi, or } \\
\text { Vital Energy }\end{array}$ & $\begin{array}{l}\text { Yang Qi - } \\
\text { La culture } \\
\text { de l'esprit } \\
\text { littéraire }\end{array}$ \\
\hline 43 & 附會 & $\begin{array}{l}\text { Aggregare } \\
\text { e combinare }\end{array}$ & $\begin{array}{l}\text { Organization } \\
\quad(f u-b u i)\end{array}$ & $\begin{array}{l}\text { Assemblage } \\
\text { and Collation }\end{array}$ & Organization & $\begin{array}{c}F u H u i- \\
\text { La rédaction }\end{array}$ \\
\hline 44 & 總術 & $\begin{array}{c}\text { La tecnica } \\
\text { della scrittura: } \\
\text { conclusoni }\end{array}$ & $\begin{array}{c}\text { Discussion on } \\
\text { the art } \\
\text { of writing } \\
(t s u n g-s b u)\end{array}$ & Methodology & $\begin{array}{c}\text { Summarizing } \\
\text { the Art } \\
\text { of Writing }\end{array}$ & $\begin{array}{l}\text { Zong Shu- } \\
\text { Les techniques } \\
\text { de l'écriture }\end{array}$ \\
\hline 45 & 時序 & $\begin{array}{l}\text { I tempi } \\
\text { e le opere }\end{array}$ & $\begin{array}{c}\text { Literary } \\
\text { development } \\
\text { and time } \\
(\text { shib-bsü) }\end{array}$ & $\begin{array}{c}\text { Chronological } \\
\text { Order }\end{array}$ & $\begin{array}{c}\text { Literature } \\
\text { and the Times }\end{array}$ & $\begin{array}{c}\text { Shi Xu- } \\
\text { L'évolution } \\
\text { de l'écriture } \\
\text { avec les temps }\end{array}$ \\
\hline
\end{tabular}




\begin{tabular}{|c|c|c|c|c|c|c|}
\hline & $\begin{array}{l}\text { 文心 } \\
\text { 雕龍 }\end{array}$ & (Liu Xie 1995) & (Liu Xie 1959) & (Liu Xie 1999) & (Liu Xie 2003) & (Liu Xie 2010) \\
\hline 46 & 物色 & $\begin{array}{l}\text { I colori } \\
\text { delle cose }\end{array}$ & $\begin{array}{l}\text { The physical } \\
\text { world }\end{array}$ & $\begin{array}{l}\text { The Beauty } \\
\text { of Nature }\end{array}$ & $\begin{array}{l}\text { The Forms } \\
\text { of the Natural } \\
\text { World }\end{array}$ & $\begin{array}{c}W u S e- \\
\text { Les couleurs } \\
\text { de la nature }\end{array}$ \\
\hline 47 & 才略 & $\begin{array}{c}\text { Sillabo } \\
\text { degli scrittori } \\
\text { più insigni }\end{array}$ & $\begin{array}{l}\text { Literary } \\
\text { talents }\end{array}$ & Talent & $\begin{array}{l}\text { Literary } \\
\text { Talents }\end{array}$ & $\begin{array}{l}\text { Cai Lüe- } \\
\text { Les aptitudes } \\
\text { des écrivains }\end{array}$ \\
\hline 48 & 知音 & $\begin{array}{l}\text { Conoscere } \\
\text { i suoni }\end{array}$ & $\begin{array}{c}\text { An } \\
\text { understanding } \\
\text { critic } \\
(\text { cbib-yin })\end{array}$ & $\begin{array}{l}\text { Understanding } \\
\text { Another's Voice }\end{array}$ & $\begin{array}{c}\text { An } \\
\text { Appreciative } \\
\text { Critic }\end{array}$ & $\begin{array}{c}\text { Zhi Yin- } \\
\text { L'appréciation } \\
\text { littéraire }\end{array}$ \\
\hline 49 & 程器 & $\begin{array}{l}\text { La capacità } \\
\text { e il recipiente }\end{array}$ & 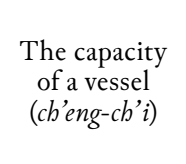 & $\begin{array}{l}\text { Weighing } \\
\text { the Vessel }\end{array}$ & $\begin{array}{c}\text { Moral } \\
\text { Integrity }\end{array}$ & $\begin{array}{c}\text { Cheng } Q i \text { - } \\
\text { La réalisation } \\
\text { de la vertu } \\
\text { et de la morale } \\
\text { chez les auteurs }\end{array}$ \\
\hline 50 & 序志 & $\begin{array}{l}\text { Disegno } \\
\text { dell'opera }\end{array}$ & ----- & $\begin{array}{l}\text { Declaration } \\
\text { of Intent }\end{array}$ & $\begin{array}{l}\text { My Intentions, } \\
\text { or Postscript }\end{array}$ & $\begin{array}{c}X u Z h i- \\
\text { La postface }\end{array}$ \\
\hline
\end{tabular}

\subsection{The architecture of the titles}

The table above may be observed from many and various points of view: first, it is worth to briefly commenting on the quantitative profile. As can be seen, the amount of information disclosed in the translation - obtained from the rendering of only two characters - appears to be a variable factor, both within each version and in the comparison of the versions listed in rows. Due to the extreme semantic density and conciseness of the Chinese characters - almost unattainable by European languages - every part had to be untied in its rhythm, form and meaning. Concerning rhythm and form, we may notice general tendencies, instead of rigorous patterns, adopted by the above-mentioned translators. To put it more specifically, more than the others, Lavagnino's version (Liu Xie 1995) together with the one by Wong, Lo, Lam (Liu Xie 1999), seemilgly tend to preserve the shape of the composition, that is, the binomial style and an essential language, while Yang Guobin (Liu Xie 2003) seems to prefer a richer wording to rephrase the concepts. Shih (Liu Xie 1959) often resorts to the Romanized transcription of the Chinese characters, enclosed by brackets after the translation, whereas Chen Shuyu (Liu Xie 2010) methodologically puts the pinyin before the French translation, separated by a hyphen. 
An interesting example of this situation can be drawn from the comparison of the titles given to chapter 7, Yuefu 樂府 (see Tab. 2).

Table 2.

\begin{tabular}{|c|c|c|c|c|c|}
\hline 7 & Yue fu & $\begin{array}{c}\text { Musical poetry } \\
(y \ddot{u} e b-f u)\end{array}$ & $\begin{array}{c}\text { The Yuefu } \\
\text { Poems }\end{array}$ & $\begin{array}{c}Y u e f u, \\
\text { or Poems } \\
\text { of the "Music } \\
\text { Bureau" }\end{array}$ & $\begin{array}{c}Y u e F u- \\
\text { Le poème } \\
\text { chanté }\end{array}$ \\
\hline
\end{tabular}

\subsection{Semantic patterning}

The latter example gives input to further considerations. Given that absolute equivalence between different languages may be considered a utopic goal, the translators have devised different strategies to re-code the meaning within the characters. One of them (see Tabs. 2-5) is the transcription of the characters in the title, through Romanization or pinyin, when a comparable concept cannot be found in the receiving language and culture, as the case of literary genres.

Table 3.

\begin{tabular}{|c|c|c|c|c|c|c|}
\hline 5 & $\begin{array}{c}\text { I giudizi } \\
\text { sul “Li Sao" }\end{array}$ & $\begin{array}{c}\text { An analysis } \\
\text { of Sao }\end{array}$ & $\begin{array}{c}\text { Isolating } \\
\text { Sao Poetry }\end{array}$ & $\begin{array}{c}\text { Evaluating Sao, } \\
\text { or the Songs } \\
\text { of the South }\end{array}$ & $\begin{array}{c}\text { Bian Sao- } \\
\text { L'évaluation } \\
\text { des élégies } \\
\text { de Chu }\end{array}$ \\
\hline
\end{tabular}

And also:

Table 4.

\begin{tabular}{|c|c|c|c|c|c|c|}
\hline 8 & 銓賦 & $\begin{array}{c}\text { La verità } \\
\text { sul } f u\end{array}$ & $\begin{array}{c}\text { Elucidation } \\
\text { of } f u\end{array}$ & $\begin{array}{c}\text { Explaining } \\
F u \text { Poetry }\end{array}$ & $\begin{array}{c}\text { Interpreting } \\
F u, \text { or } \\
\text { Rhyme-prose }\end{array}$ & $\begin{array}{c}\text { Quan Fu - } \\
\text { Linterprétation } \\
\text { du } f u\end{array}$ \\
\hline
\end{tabular}

Table 5.

\begin{tabular}{|c|c|c|c|c|c|c|}
\hline 9 & 頌讚 & Song e zan & $\begin{array}{c}\text { Ode and } \\
\text { Pronouncement } \\
\text { (the sung } \\
\text { and the tsan) }\end{array}$ & $\begin{array}{c}\text { Eulogistic } \\
\text { Songs } \\
\text { and Summaries }\end{array}$ & $\begin{array}{c}\text { Hymn } \\
\text { and Eulogy }\end{array}$ & $\begin{array}{c}\text { Song Zan - } \\
\text { Léloge } \\
\text { et le conseil }\end{array}$ \\
\hline
\end{tabular}

Furthermore, there are situations the title appears only partially translated from Chinese (see Tabs. 6-8), that may be explained by the semantic displacement. 
Table 6.

\begin{tabular}{|c|c|c|c|c|c|c|}
\hline 18 & $\begin{array}{c}\text { Treatise } \\
\text { and } \\
\text { Discussion } \\
\text { (the lun } \\
\text { and the } \text { shuo) }\end{array}$ & $\begin{array}{c}\text { Argument } \\
\text { and } \\
\text { Persuasion } \\
\text { e onorazione }\end{array}$ & $\begin{array}{c}\text { The Treatise } \\
\text { and } \\
\text { the Speech }\end{array}$ & $\begin{array}{c}\text { Lun Shuo- } \\
\text { Les essais } \\
\text { et les discours }\end{array}$ \\
\hline
\end{tabular}

Table 7.

\begin{tabular}{|c|c|c|c|c|c|c|}
\hline 21 & $\begin{array}{c}\text { Sacrifici } \\
\text { al Cielo } \\
\text { e sacrifici } \\
\text { alla Terra }\end{array}$ & $\begin{array}{c}\text { Sacrifices } \\
\text { to Heave } \\
\text { and Earth } \\
\text { (feng shan) }\end{array}$ & $\begin{array}{c}\text { The Fengshan } \\
\text { Sacrifice }\end{array}$ & $\begin{array}{c}\text { The Sacrificial } \\
\text { Address } \\
\text { to Heaven } \\
\text { and Earth }\end{array}$ & $\begin{array}{c}\text { Feng Shan - } \\
\text { Les écrits } \\
\text { des sacrifices } \\
\text { au Ciel } \\
\text { et à la Terre }\end{array}$ \\
\hline
\end{tabular}

Table 8 .

\begin{tabular}{|l|c|c|c|c|c|c|}
\hline 36 & 比興 & $\begin{array}{c}\text { Paragone } \\
\text { e xing }\end{array}$ & $\begin{array}{c}\text { Metaphor and } \\
\text { Allegory } \\
\text { (pi and bsing) }\end{array}$ & $\begin{array}{c}\text { Bi and Xing - } \\
\text { Two Types } \\
\text { of Metaphor }\end{array}$ & $\begin{array}{c}\text { Comparison } \\
\text { and Metaphor }\end{array}$ & $\begin{array}{c}\text { Bi Xing - } \\
\text { ea comparaison } \\
\text { et la métaphore }\end{array}$ \\
\hline
\end{tabular}

This happens not only with literary genres, but also with concepts that don't meet any correspondence outside of Chinese language and culture, and that deserve complex explanations into European languages. This is the case of Dao (see Tab. 9) in the first chapter, and of $q i$ 氣, in chapter 42 (see Tab. 10).

Table 9.

\begin{tabular}{|c|c|c|c|c|c|c|}
\hline 1 & 原道 & $\begin{array}{c}\text { L'Origine } \\
\text { nel Dao }\end{array}$ & $\begin{array}{c}\text { On Tao, } \\
\text { the source }\end{array}$ & $\begin{array}{c}\text { The Way } \\
\text { the Origin }\end{array}$ & $\begin{array}{c}\text { Tracing } \\
\text { the Origin } \\
\text { to the Dao }\end{array}$ & $\begin{array}{c}\text { Yuan Dao- } \\
\text { Lorigine } \\
\text { de l'écriture }\end{array}$ \\
\hline
\end{tabular}

Table 10.

\begin{tabular}{|c|c|c|c|c|c|c|}
\hline 42 & $\begin{array}{c}\text { Come } \\
\text { alimentare } \\
\text { il } q i\end{array}$ & $\begin{array}{c}\text { The nourishing } \\
\text { of vitality }\end{array}$ & $\begin{array}{c}\text { Preservation } \\
\text { of the } \\
\text { Life-breath }\end{array}$ & $\begin{array}{c}\text { Nourishing } \\
q i \text {, or } \\
\text { Vital Energy }\end{array}$ & $\begin{array}{c}\text { Yang } Q i- \\
\text { La culture } \\
\text { de l'esprit } \\
\text { littéraire }\end{array}$ \\
\hline
\end{tabular}

In the first example, three translations out of five do not disclose the culture-specific concept of Dao 道 (or Tao), subject of extensive investigations by literary critics, and a term the public likely have already become acquainted with. It is relevant that Wong, Lo, Lam (Liu Xie 1999) decided to be more explicit by rephrasing Dao into "The Way", 
in English: the broad distance between the solutions reveal the intrinsic polysemy of the word Dao 道. A similar circumstance occurs with the term qi 氣 (see Tab. 10). The complexity of the meaning behind the word can hardly be resolved in a single corresponding expression, therefore, three different strategies can be identified. The first, adopted by Lavagnino (Liu Xie 1995), is the transcription/rewriting of the Chinese word $q i$ 氣 through the pinyin. The second is the addition of a concise explanation, as demonstrated by Yang Guobin's version (Liu Xie 2003), where $q i$ is followed by "or Vital Energy". The same pattern is also applied to the above-mentioned chapter 7 ("Yuefu, or Poems of the 'Music Bureau") and to the following case, with the expression Shen si 神思 (see Tab. 11).

Table 11 .

\begin{tabular}{|c|c|c|c|c|c|c|}
\hline 26 & 神思 & $\begin{array}{c}\text { Il pensiero } \\
\text { poetico }\end{array}$ & $\begin{array}{c}\text { Spiritual } \\
\text { thought } \\
\text { or Imagination } \\
(s h e n-s s u)\end{array}$ & $\begin{array}{c}\text { Magical } \\
\text { Imagination }\end{array}$ & $\begin{array}{c}\text { Shensi, } \\
\text { or Imagination }\end{array}$ & $\begin{array}{c}\text { Lhen }- \\
\text { La pensée } \\
\text { et } \\
\text { l'imagination }\end{array}$ \\
\hline
\end{tabular}

The third solution corresponds to the full translation of the term and the omission of the Chinese: qi becomes "vitality" (Liu Xie 1959), "lifebreath" (Liu Xie 1999) and "esprit littéraire" (Liu Xie 2010). While the English translators found similar - but not identical - solutions, the French translator Chen Shuyu decided to interpret Yang 養 ("alimentare") "Nourishing"/"Preservation") as the broader concept of "La culture", that may only be inferred from the chapter's context. The latter could be an example of what is called "domesticated translation" (Venuti 1995) - that tends to smooth over the linguistic and cultural gap - whereas the first may be seen as a "foreignized translation" (ibid.), that prefers to be faithful to the source text and to preserve its linguistic and cultural flavor. To this respect, it is useful to mention other specific examples marking the difference between domesticated and foreignized translation.

With regard to domestication, on the one side, it may be noticed Shih's tendency to prosaically reformulate binomials expressing metaphors (see Tabs. 12-13).

Table 12 .

\begin{tabular}{|c|c|c|c|c|c|c|}
\hline 37 & 誇飾 & L'iperbole & $\begin{array}{c}\text { Embellishment } \\
\text { as description }\end{array}$ & Hyperbole & Hyperbole & $\begin{array}{c}\text { Kua Shi- } \\
\text { L'hyperbole }\end{array}$ \\
\hline
\end{tabular}


Table 13.

\begin{tabular}{|l|l|l|c|c|c|c|}
\hline 29 & 通變 & $\begin{array}{c}\text { Flexible } \\
\text { Continuità e } \\
\text { mutamento }\end{array}$ & $\begin{array}{c}\text { adaptability } \\
\text { to varying } \\
\text { situations } \\
\text { (t'ung-pien) }\end{array}$ & $\begin{array}{c}\text { Continuity } \\
\text { and Change }\end{array}$ & $\begin{array}{c}\text { Continuity } \\
\text { and Change }\end{array}$ & $\begin{array}{c}\text { Tong Bian- } \\
\text { Comprendre } \\
\text { le changement }\end{array}$ \\
\hline
\end{tabular}

And moreover, Chen Shuyu's solutions seem to reveal the author's inclination to dissipate interpretative ambiguities (see Tab. 14, and again Tab. 7).

Table 14

\begin{tabular}{|l|c|c|c|c|c|}
\hline 49 & 程器 & $\begin{array}{c}\text { La capacità } \\
\text { e il recipiente }\end{array}$ & $\begin{array}{c}\text { The capacity } \\
\text { of a vessel } \\
(\text { ch'eng-ch'i) }\end{array}$ & $\begin{array}{c}\text { Weighing } \\
\text { the Vessel }\end{array}$ & $\begin{array}{c}\text { Cheng } Q i- \\
\text { Moral } \\
\text { Integrity } \\
\text { déalisation }\end{array}$ \\
$\begin{array}{c}\text { de la vertu } \\
\text { et de la morale } \\
\text { chez les auteurs }\end{array}$ \\
\hline
\end{tabular}

And sometimes, to clarify implicit meanings (see Tabs. 15-16).

Table 15

\begin{tabular}{|l|l|c|c|c|c|c|}
\hline 32 & $\begin{array}{c}\text { Gettare } \\
\text { in forma } \\
\text { e tagliare }\end{array}$ & $\begin{array}{c}\text { Casting } \\
\text { and cutting, } \\
\text { or, on editing } \\
\text { of ideas } \\
\text { and rhetoric } \\
\text { (jung-ts'ai) }\end{array}$ & $\begin{array}{c}\text { Modelling } \\
\text { and Tailoring }\end{array}$ & $\begin{array}{c}\text { Casting } \\
\text { and Cutting }\end{array}$ & $\begin{array}{c}\text { Rong Cai- } \\
\text { Les détails } \\
\text { et la concision }\end{array}$ \\
\hline
\end{tabular}

Table 16.

\begin{tabular}{|c|c|c|c|c|c|c|}
\hline 45 & 時序 & $\begin{array}{c}\text { I tempi } \\
\text { e le opere }\end{array}$ & $\begin{array}{c}\text { Literary } \\
\text { development } \\
\text { and time } \\
(s h i b-b s \ddot{u})\end{array}$ & $\begin{array}{c}\text { Chronological } \\
\text { Order }\end{array}$ & $\begin{array}{c}\text { Literature } \\
\text { and the Times }\end{array}$ & $\begin{array}{c}\text { L'évolution } \\
\text { de l'écriture } \\
\text { avec les temps }\end{array}$ \\
\hline
\end{tabular}

On the other side, Lavagnino (Liu Xie 1995) and Yang Guobin (Liu Xie 2003) give an example of what can be seen as preservation of the semantic patterning by underlining in quotation marks foreign and foreignizing Liu Xie's expressions (see Tab. 17).

Table 17.

\begin{tabular}{|c|c|c|c|c|c|c|}
\hline 28 & 風骨 & $\begin{array}{c}\text { "Vento" } \\
\text { e "ossa" }\end{array}$ & $\begin{array}{c}\text { The wind } \\
\text { and the bone } \\
(f e n g-k u)\end{array}$ & $\begin{array}{c}\text { The Affective } \\
\text { Air } \\
\text { and the } \\
\text { Literary Bones }\end{array}$ & $\begin{array}{c}\text { "Wind" } \\
\text { and "Bone" }\end{array}$ & $\begin{array}{c}\text { Feng } G u- \\
\text { Le charme } \\
\text { et l'essence }\end{array}$ \\
\hline
\end{tabular}




\subsection{Lexical choices}

Another interesting aspect of the comparative analysis, directly linked to abovementioned cases, is observation of the semantic cohesion through lexical items. Generally speaking, it can be said that the five versions seem to be convergent. Nonetheless, considering the diverse production contexts and the constitutive differences of the target languages, it may be not particularly surprising that the translators adopted differing solutions for specific terms, as we could see in relation to the transcription or rephrasing of culture-specific concepts and Chinese terms. The following tables offer several examples of "diffractions" or lexical variations (see Tabs. 18-20).

Table 18

\begin{tabular}{|l|c|c|c|c|c|c|}
\hline 4 & 正緯 & $\begin{array}{c}\text { Corretta lettura } \\
\text { degli Apocrifi }\end{array}$ & $\begin{array}{c}\text { Emendation } \\
\text { of Apocrypha }\end{array}$ & $\begin{array}{c}\text { Putting } \\
\text { the Cabala } \\
\text { in Order }\end{array}$ & $\begin{array}{c}\text { A Proper } \\
\text { Understanding } \\
\text { of Apocrypha }\end{array}$ & $\begin{array}{c}\text { Zheng Wei- } \\
\text { La correction } \\
\text { des apocryphes }\end{array}$ \\
\hline
\end{tabular}

It is to a certain extent curious the choice made by Wong, Lo and Lam (Liu Xie 1999), when replacing Wei 緯 - commonly translated with "Apocrypha" - with the term "Cabala" that is strongly connotated in cultural terms and that immediately evokes the Kabbalah of the Hebrew mystic tradition.

Table 19

\begin{tabular}{|c|c|c|c|c|c|c|}
\hline 40 & 隱秀 & $\begin{array}{c}\text { Il recondito } \\
\text { e l'eccellente }\end{array}$ & $\begin{array}{c}\text { The recondite } \\
\text { and } \\
\text { the conspicuous } \\
(y i n-b s i u)\end{array}$ & $\begin{array}{c}\text { Hidden Grace } \\
\text { and the Visible } \\
\text { Flower }\end{array}$ & $\begin{array}{c}\text { Concealed } \\
\text { and Evident } \\
\text { Excellence }\end{array}$ & $\begin{array}{c}\text { Yin Xiu - } \\
\text { Limplicite } \\
\text { et l'aphorisme }\end{array}$ \\
\hline
\end{tabular}

Something similar happens in chapter 49 , where the translators re-interpret the Chinese title (Yin xiu 隱秀) anticipating the metaphoric reference to the blossoming of the poetic composition, illustrated within the chapter.

Table 20.

\begin{tabular}{|c|c|c|c|c|c|c|}
\hline 31 & 情采 & $\begin{array}{c}\text { Emozioni } \\
\text { e bellezza }\end{array}$ & $\begin{array}{c}\text { Emotion } \\
\text { and Literary } \\
\text { expression }\end{array}$ & $\begin{array}{c}\text { Substance } \\
\text { and Style }\end{array}$ & $\begin{array}{c}\text { Feeling } \\
\text { and Art }\end{array}$ & $\begin{array}{c}\text { Qing Cai - } \\
\text { les exprestions et } \\
\text { littéraires }\end{array}$ \\
\hline
\end{tabular}


Another relevant example is offered by chapter 31 (Qing cai 情采), where there are significant discrepancies in the interpretation of the two Chinese terms qing 情 and cai 采 ${ }^{4}$. Qing 情 is translated as "Emotions" (Liu Xie 1995; 1959; 2010) or "Feeling" (Liu Xie 2003), and even "Substance" (Liu Xie 1999). Conversely, cai 采 gives rise to unparalleled interpretations, that are "bellezza" (beauty) (Liu Xie 1995), "Literary expressions" / “expressions littéraires" (Liu Xie 1959; 2010), "Style" (Liu Xie 1999) and "Art" (Liu Xie 2003). This could stand as another exemplary case of the inherent polysemy and the semantic unattainable richness possessed by the Chinese binomial title.

\section{Concluding REMARKS}

While comparing the Chinese original with different and differing translations into three European languages, two aspects should be borne in mind. Firstly, the theoretic and aesthetic preciousness of Liu Xie's masterpiece, whose magnificently "carved" language treasures an invaluable semantic richness. This makes the Wenxin diaolong a unique work in the world's literary history. Secondly, the unbridgeable gap between languages and cultures, and especially between the classical Chinese and contemporary European alphabetic languages, what brings to the point of the gains and losses in translation process. As Lawrence Venuti stated:

Both foreign text and translation are derivative: both consist of diverse linguistic and cultural materials that neither the foreign writer nor the translator originates, and that destabilize the work of signification, inevitably exceeding and possibly conflicting with their intentions. As a result, a foreign text is the site of many different semantic possibilities that are fixed only provisionally in any one translation, on the basis of varying cultural assumptions and interpretive choices, in specific social situations, in different historical periods. Meaning is a plural and contingent relation, not an unchanging unified essence, and therefore a translation cannot be judged according to mathematics-based concepts of semantic equivalence or one-to-one correspondence. [...] The viability of a translation is established by its relationship to the cultural and social conditions under which it is produced and read. (Venuti 1995, 18)

4 Except from the strong resemblance of the French version by Chen Shuyu (Liu Xie 2010) to the English version by Shih (Liu Xie 1959). 
In the light of this, each translation strategy concretely represents the translator's substantial effort to disclose the meaning beyond the words. Therefore, their inconsistencies add value to the to the original work, by giving prominence to the intrinsic polysemy of the text.

\section{REFERENCES}

Bassnett, Susan. 2002. Translation Studies. 3rd edn. London - New York: Routledge. Berman, Antoine. 1995. L'épreuve de l'étranger. Paris: Gallimard.

Jakobson, Roman. 1959. “On Linguistic Aspects of Translation”. In On Translation, edited by Reuben A. Brower, 232-239. Cambridge: Harvard University Press.

Liu Xie. 1959. The Literary Mind and the Carving of Dragons: a Study of Thought and Pattern in Chinese Literature. New York: Columbia University Press [Eng. transl. Vincent Yu-chung Shih].

Liu Xie. 1995. Il tesoro delle Lettere. Un intaglio di draghi. Milano: Luni Editrice [trad. it. Alessandra C. Lavagnino].

Liu Xie, 1999. The Book of Literary Design. Hong Kong: Hong Kong University Press [Eng. transl. Wong Siu-Kit, Chung-hang Lo, Allan, and Lam Kwong-tai].

Liu Xie. 2003. Dragon-Carving and the Literary Mind. Beijing: Foreign Language Teaching and Research Press [Eng. transl. Yang Guobin].

Liu Xie. 2010. L'essence de la littérature et la gravure des dragons. Beijing: Éditions en Langues étrangères [trad. fr. Chen Shuyu].

Nida, Eugene. 2012. "Chapter 12: Principle of Correspondence". In The Translation Studies Reader. 3rd edn., edited by Lawrence Venuti, 141-155. London - New York: Routledge.

Ricoeur, Paul. 2004. Sur la traduction. Paris: Bayard.

Steiner, George. 1975. After Babel. London: Oxford University Press.

Vandermeersch, Léon. 2013. Les deux raisons de la pensée chinoise. Divination et idéographie. Paris: Gallimard.

Venuti, Lawrence. 1995. The Translator's Invisibility: A History of Translation. London New York: Routledge. 
